\title{
Comparison of the effects of carvedilol and nebivolol on diastolic functions of the left ventricle in patients with non-ischemic heart failure
}

\author{
Abdullah Dogan ${ }^{1}$, Mustafa Karabacak², Şenol Tayyar ${ }^{3}$, \\ Dogan Erdogan ${ }^{1}$, Mehmet Ozaydin ${ }^{1}$ \\ ${ }^{1}$ Department of Cardiology, Medical Faculty, Suleyman Demirel University, Isparta, Turkey \\ ${ }^{2}$ Physician in Cardiology, Medical Faculty, Suleyman Demirel University, Isparta, Turkey \\ ${ }^{3}$ Pysician in Cardiology, Agrı State Hospital, Agrı, Turkey.
}

\begin{abstract}
Background: We investigated whether carvediolol or nebiovolol with vasodilator properties will produce different effects on diastolic function of the left ventricle (LV) in heart failure (HF) with low ejection fraction (EF).
\end{abstract}

Methods: Sixty-one non-ischemic HF patients with $E F \leq 40 \%$ randomly received carvedilol $(n=31,16$ male) or nebivolol $(n=30,19$ male). Clinical and echocardiographic evaluations were performed at baseline, 3 and 6 months after therapy. Mitral inflow velocities ( $E$ and A waves), deceleration time of $E$ wave (DT), isovolumetric relaxation time (IVRT), mitral annular velocities (Ea and $A$ a waves) were evaluated. Mitral $E / A$ and $E / E$ a ratios were calculated. Results: In carvediolol and nebivolol groups, mitral E/A ratio (from $1.08 \pm 0.31$ to $0.87 \pm$ \pm 0.30 vs. from $0.98 \pm 0.20$ to $0.80 \pm 0.20, p=0.30$ ) and IVRT (from $108 \pm 13$ to $94 \pm$ $\pm 10 \mathrm{~ms} v \mathrm{vs}$. from $107 \pm 22$ to $92 \pm 10 \mathrm{~ms}, p=0.25)$ similarly decreased while DT prolonged (from $184 \pm 40$ to $218 \pm 42 \mathrm{~ms}$ vs. from $193 \pm 37$ to $222 \pm 36 \mathrm{~ms}, p=0.71$ ). Also, $E / E a$ ratio significantly decreased in each group $(p=0.01)$, but it was lower in nebivolol group than carvedilol group at 6 months $(10.2 \pm 2$ vs. $11.8 \pm 2, p=0.01)$. Carvediolol and nebivolol reduced similarly $N$-terminal pro-B type natriuretic peptide level (from 666 to 137 vs. 661 to $123 \mathrm{pg} / \mathrm{dL}, p=0.41$, respectively) and improved functional capacity ( $p>0.05)$.

Conclusions: At 6 month follow-up, carvedilol and nebivolol appear to similarly improve LV diastolic functions in non-ischemic HF patients. (Cardiol J 2014; 21, 1: 76-82)

Key words: carvedilol, nebivolol, diastolic function, heart failure

\section{Introduction}

Despite therapeutic advances, heart failure (HF) is a disabling disease with poor prognosis [1]. Beta-blockers are cornerstone agents in HF therapy because they prevent the deleterious effects of activation of sympathetic nervous system $[1,2]$. Diastolic abnormalities of the left ventricle (LV) are important determinants for exercise intolerance in HF patients [3, 4].

Carvedilol and nebiovolol are new-generation agents with favourable vasodilator and/or antioxi-

Address for correspondence: Abdullah Dogan, MD, Department of Cardiology, Medical Faculty, Suleyman Demirel University, Isparta, Gazi Kemal Mah 1309 Sokak, Sarac Sitesi C blok No:9/5 32100, Turkey, fax: +90 2462371758 ,

e-mail: adogan35@hotmail.com

Received: 22.03.2013 Accepted: 15.05.2013 
dant properties, and they are used for chronic $\mathrm{HF}$ treatment $[1,5,6]$. They can reverse cardiac remodeling resulting from sympathetic overactivation in HF patients [7-11]. Moreover, they improve symptoms and survival in HF patients [9, 11-14]. However, there is uncertainity whether their fovarable effects will similarly produce clinical benefit, and carvediolol may be preferred to nebivolol in HF patients [15].

On the other hand, 3 previous studies compared the effects of these 2 agents on LV functions in HF patients [9-11]. They have provided divergent results about LV diastolic functions at 6- to 24-month follow-up. In addition, transmitral to mitral annular early diastolic velocity ratio $(\mathrm{E} / \mathrm{Ea})$, a sensitive marker for high diastolic filling pressure [16] has not been evaluated in those studies. Thus, we aimed to compare the effects of carvedilol and nebivolol on $\mathrm{LV}$ diastolic functions and $\mathrm{N}$-terminal pro-B-type natriuretic peptide (NT-proBNP) level in non-ischemic $\mathrm{HF}$ patients with reduced systolic function.

\section{Methods}

\section{Patients and dose titration of study drugs}

This study included 68 consecutive patients with moderate or severe symtomatic HF (New York Heart Association [NYHA] class II to III) who had angiographically normal coronary arteries. However, 7 patients were excluded from the study because of recent atrial fibrillation $(n=2)$, beta-blocker intolerance $(n=2)$, NYHA class IV $\mathrm{HF}$ at the time of randomization $(\mathrm{n}=1)$ and refusal to repeat examinations $(n=2)$. Finally, the remaining 61 patients were randomly assigned to receive carvedilol $(\mathrm{n}=31)$ or nebivolol $(\mathrm{n}=30)$ in a single-blind and open-label fashion. They had LV ejection fraction $(\mathrm{EF}) \leq 40 \%$ in previous 3 months. The patients who had the following features were not considered for inclusion into this study at the beginning. These features were $\mathrm{HF}$ with significant coronary stenosis, history of myocardial infarction, moderate or severe valvular heart disease, rest heart rate $<60 \mathrm{bpm}$, systolic blood pressure (SBP) $<100 \mathrm{~mm} \mathrm{Hg}$, history of asthma or use of bronchodilators, hypo- or hyperthyroidism, hepatic or renal failure (serum creatinin $>2.0 \mathrm{mg} / \mathrm{dL}$ ), rhythm disturbances including second or third degree heart block, sick sinus syndrome, atrial fibrillation, and complete bundle branch block.

In patients who were on beta-blocker therapy before the study, beta-blocker therapy was stopped at least 1 week for drug elimination. Thereafter, carvedilol was started at $3.125 \mathrm{mg}$ twice da- ily and then up-titrated to $6.25,12.5$ and $25 \mathrm{mg}$ (target dose) twice a day at 3 to 5-day intervals if the previous dose was tolerated. Similarly, nebivolol was given at $1.25 \mathrm{mg}$ once a day and then up-titrated to 2.5, 5 and $10 \mathrm{mg}$ (target dose) once a day if the previous dose was tolerated. When the up-titration dose was not tolerated, the previous tolerable dose was considered maximum tolerable dose for each agent. The patient's tolerance was evaluated according to the following criteria; resting heart rate $>55 \mathrm{bpm}, \mathrm{SBP} \geq 100 \mathrm{~mm} \mathrm{Hg}$, no drop in SBP $<30 \mathrm{~mm} \mathrm{Hg}$ on standing position, no new symptoms of dizziness, or dyspnea. After the target or maximum tolerable dose, patients were evaluated in an out-patient clinic at $1^{\text {st }}, 3^{\text {rd }}$ and $6^{\text {th }}$ month. Complete blood count, biochemical analysis and echocardiographic measurements were made in patients at baseline, 3 and 6 months after target dose. Heart rate, BP and body weight were recored at each visit. Also, functional status of patients was assessed according to NYHA. Other medications were prescribed according to current chronic HF guideline [1]. All patients received angiotensin-converting enzyme inhibitor (ACEI, lisinopiril) and diuretics in appropriate doses. Candesartan, angiotensin receptor blockers (ARB) were given when ACEI intolerance occured.

\section{Blood sampling and assays}

Blood samples were drawn for routine hematological and biochemical analyses. Biochemical analyses were preformed with "Olympus AU-640". For NT-proBNP assays, a 5-mL blood sample was collected into a plastic tube containing potassium EDTA. Plasma level of NT-proBNP was measured by using an electrochemiluminiscence immunoassay with Elecsys 2010 analyser (Roche Diagnostics, Mannheim, Germany).

\section{Echocardiography}

Echocardiographic examinations were performed by the same investigator (MK) who blinded to the patients' data at baseline, 3 and 6 months. Measurements were acquired at the end of expiration during normal breathing in the left lateral decubitus position. Two-dimensional, M-mode, and Doppler echocardiographic measurements were obtained according to the recommendations of the American Society of Echocardiography with System 5 Echocardiography Machine (GE Vingmed Ultrasound) with a $2.5 \mathrm{MHz}$ transducer [17]. The mean of 3 cardiac cycles with ECG record was evaluated for measurements. The left atrial size, LV diameters and wall thickness were measured by using M-mode echocardiography. LVEF was calculated by Simpson's method. 
Table 1. Demographic, clinical and laboratory characteristics of carvediolol and nebivolol groups.

\begin{tabular}{|c|c|c|c|}
\hline & Carvedilol group $(n=31)$ & Nebivolol group $(n=30)$ & $\mathbf{P}$ \\
\hline Mean age [year] & $61 \pm 11$ & $60 \pm 14$ & 0.73 \\
\hline Male/female & $16 / 15$ & $19 / 11$ & 0.36 \\
\hline Body weight [kg] & $78 \pm 18$ & $75 \pm 12$ & 0.48 \\
\hline NYHA class II/III & $52 \% / 48 \%$ & $60 \% / 40 \%$ & 0.61 \\
\hline Current smoker & $8(26 \%)$ & $11(37 \%)$ & 0.42 \\
\hline Hypertension & $15(45 \%)$ & $15(48 \%)$ & 0.90 \\
\hline Diabetes mellitus & $5(16 \%)$ & $5(17 \%)$ & 0.99 \\
\hline Hyperlipidemia & $7(22 \%)$ & $10(33 \%)$ & 0.40 \\
\hline Obesity (BMI $\geq 30 \mathrm{~kg} / \mathrm{m}^{2}$ ) & $11(35 \%)$ & $8(26 \%)$ & 0.46 \\
\hline Systolic BP [mm Hg] & $143 \pm 17$ & $141 \pm 13$ & 0.57 \\
\hline Diastolic BP [mm Hg] & $91 \pm 15$ & $90 \pm 10$ & 0.60 \\
\hline Heart rate [bpm] & $81 \pm 9$ & $82 \pm 9$ & 0.72 \\
\hline \multicolumn{4}{|l|}{ Medications: } \\
\hline ACEI/ARB & $29(93 \%) / 2(7 \%)$ & $28(93 \%) / 2(7 \%)$ & 0.96 \\
\hline Spirinolacton & $9(29 \%)$ & $5(17 \%)$ & 0.25 \\
\hline Other diuretics & $31(100 \%)$ & $30(100 \%)$ & 0.99 \\
\hline Statins & $4(13 \%)$ & $2(7 \%)$ & 0.67 \\
\hline Digoxin & $2(6 \%)$ & $2(7 \%)$ & 0.97 \\
\hline Aspirin & $20(64 \%)$ & $11(36 \%)$ & 0.03 \\
\hline Creatinine [mg/dL] & $0.97 \pm 0.2$ & $1.0 \pm 0.3$ & 0.27 \\
\hline Sodium [mEq/L] & $140 \pm 3.3$ & $141 \pm 3.6$ & 0.27 \\
\hline Potassium [mEq/L] & $4.4 \pm 0.4$ & $4.4 \pm 0.4$ & 0.84 \\
\hline Hematocrit [\%] & $42 \pm 4.7$ & $42 \pm 4.8$ & 0.72 \\
\hline NT-proBNP [pg/mL] & 666 (442-1350) & 661 (455-1013) & 0.61 \\
\hline
\end{tabular}

ACEI — angiotensin converting enzyme inhibitor; ARB — angiotensin-1 receptor blockers; BMI — body mass index; BP — blood pressure; NYHA - New York Heart Association; NT-proBNP — N-terminal pro-B-type natriuretic peptide

The transmitral flow was recorded from the apical 4-chamber view with the pulsed Doppler. Sample volume was placed at the tips of the mitral leaflet during diastole. The following variables were measured from transmitral flow: peak early diastolic (E) wave velocity, late diastolic (A) wave velocity, $\mathrm{E} / \mathrm{A}$ ratio, and $\mathrm{E}$ wave deceleration time (DT). Isovolumetric relaxation time (IVRT) was calculated in apical 5-chamber view [17].

Pulsed Doppler tissue images were obtained from the apical 4 -chamber view with minimum frame rates of 100/s. At least 3 consecutive traces of septal and lateral segments of mitral annulus were recorded. Their peak early (Ea-wave) and late diastolic velocity (Aa-wave) were measured and the $\mathrm{Ea} / \mathrm{Aa}$ ratio was estimated. The average of septal and lateral annular velocities was calculated and considered the final mitral annular velocity. Therafter, $\mathrm{E} /$ Ea ratio was calculated. With some measurements, intraobserver variabilities were $5.2 \%, 7.2 \%$ and $3.8 \%$ for EF, IVRT and E/Ea, respectively.

The study was approved by the institutional ethics committee and informed consent was obtained from each patient.

\section{Statistical analyses}

All analyses were performed with commercially available statistical program (SPSS Version 13.0, SPSS Inc., Chicago, IL). Continous variables were presented as mean \pm standard deviation and categoric ones as percentage (\%). The two study groups were compared using Student-t test or Mann-Whitney U and $\chi^{2}$ or Fisher exact test as appropriate. In each group, follow-up comparisons (baseline and 6 months) were performed by using Paired t-test and Wilcoxon ranked tests as appropriate. A 2-tailed $\mathrm{p}$ value of $<0.05$ was considered statistically significant.

\section{Results}

Baseline demographic and clinical features except the use of aspirine were comparable in carvedilol and nebiovolol groups (Table 1). Laboratory tests and initial echocardiographic measurements were also similar in the two groups (Tables 1,2 ). Target dose of study drugs was reached in $42 \%$ and $47 \%$ of patients in carvedilol and nebivolol groups, respectively $(\mathrm{p}=0.85)$. 
Table 2. Baseline echocardiographic parameters of carvediolol and nebivolol groups.

\begin{tabular}{lccc}
\hline & Carvedilol $(\mathbf{n}=\mathbf{3 1})$ & Nebivolol $(\mathbf{n}=\mathbf{3 0})$ & $\mathbf{P}$ \\
\hline LV diastolic diameter [mm] & $58 \pm 7$ & $57 \pm 6$ & 0.65 \\
LV systolic diameter [mm] & $46 \pm 7$ & $44 \pm 6$ & 0.22 \\
Septal thickness [mm] & $11 \pm 1.2$ & $11 \pm 1.5$ & 0.48 \\
Posterior wall thickness [mm] & $11 \pm 1.1$ & $10 \pm 1.1$ & 0.08 \\
LV ejection fraction [\%] & $33 \pm 4.2$ & $34 \pm 4.9$ & 0.22 \\
LA diameter [mm] & $43.4 \pm 4.2$ & $41.5 \pm 4.1$ & 0.68 \\
Mitral E velocity [cm/s] & $90 \pm 13$ & $86 \pm 18$ & 0.30 \\
Mitral A velocity [cm/s] & $84 \pm 24$ & $90 \pm 18$ & 0.31 \\
Mitral E/A ratio & $1.08 \pm 0.31$ & $0.98 \pm 0.26$ & 0.19 \\
Mitral DT [ms] & $184 \pm 40$ & $193 \pm 37$ & 0.33 \\
IVRT [ms] & $108 \pm 13$ & $107 \pm 22$ & 0.83 \\
Mitral Ea [cm/s] & $6.7 \pm 1$ & $6.8 \pm 2$ & 0.87 \\
Mitral Aa [cm/s] & $8.4 \pm 2$ & $8.8 \pm 2$ & 0.33 \\
Ea/Aa ratio & $0.78 \pm 0.1$ & $0.78 \pm 0.3$ & 0.98 \\
E/Ea ratio & $14.0 \pm 2.5$ & $13.1 \pm 2.9$ & 0.24 \\
\hline
\end{tabular}

$\mathrm{Aa}$ - mitral annular late diastolic velocity; DT — deceleration time of mitral E wave; Ea — mitral annular early diastolic velocity; IVRT — isovolumetric relaxation time; LA — left atrium; LV — left ventricle

Table 3 shows temporal changes in clinical and echocardiographical variables of both groups at 3 and 6 months. Heart rate, $\mathrm{BP}$ and body weight significantly decreased in each group (each $\mathrm{p}=0.01$ ), but these reductions were similar in both groups. Similarly, functional class significantly improved in each group $(\mathrm{p}=0.01)$, but was comparable in both groups ( $\mathrm{p}=0.54$, Table 3 , Fig. 1$)$. In addition, concomitant medications did not differ in both groups. Four patients received candesartan due to ACEI intolerance (2 patients in each group).

The LV diameters and EF significantly improved in each group at 6 months (Table 3 ). Compared with the carvedilol group, EF was slightly higher in the nebivolol group due to bigger reduction of LV systolic diameter at 3 months, but it was similar in the two groups at 6 months.

In each group, mitral $\mathrm{E}$ velocity and $\mathrm{E} / \mathrm{A}$ ratio significantly decreased (each $\mathrm{p}=0.01$ ) while A velocity remained unchanged (Table 3 ). However, these reductions were similar in both groups. Similarly, DT and IVRT significantly improved in each therapy group (each $\mathrm{p}<0.05$ ). However, these improvements were also comparable in the two groups at 3 and 6 months (Table 3). Mitral Ea/Aa ratio did not differ in the two groups, although Aa velocity was lower in the carvedilol group than in the nebivolol group $(\mathrm{p}=0.04)$. On the other hand, $\mathrm{E} / \mathrm{Ea}$ ratio significantly decreased in each group $(p=0.01)$, but it was lower in the nebivolol group than in the carvedilol group at 6 months $(10.2 \pm 2$ vs. $11.8 \pm 2, \mathrm{p}<0.01)$.
During 6 months, median NT-proBNP levels were significantly reduced with carvediolol or nebivolol therapy ( $p<0.001$, Table 3 ). However, this reduction was comparable in the two groups at 3 and 6 months.

At follow-up, there was no death, hospitalization for HF or discontinuation of study drugs for adverse effects. Drugs were well tolerated.

\section{Discussion}

In the present study, mitral $\mathrm{E} / \mathrm{A}$ ratio and IVRT similarly decreased in nonischemic HF patients who were treated with carvediolol or nebivolol at 6 months while DT similarly prolonged. Also, E/Ea ratio significantly decreased in each therapy group.

Clinical data have showed that carvedilol and nebivolol have beneficial effects on clinical outcomes, such as symptoms, exercise capacity and prognosis in HF patients with reduced EF [9, 11-14]. These useful effects are frequently attributed to the elevated LVEF due to reduction in LV volumes [8, 9, 11-14].

On the other hand, diastolic function is important for symptoms and prognosis in HF patients. Symptoms and functional capacity can be more related to diastolic dysfunction more than systolic dysfunction in HF patients [3, 4]. Beta-blockers can frequently improve LV diastolic function in association with an increase in $\mathrm{EF}$ of HF patients $[18,19]$. However, they can recover diastolic dysfunction even without a concomitant EF elevation 
Table 3. Temporal changes in clinical and echocardiographic variables at 3 and 6 months.

\begin{tabular}{|c|c|c|c|c|c|c|}
\hline & \multicolumn{2}{|c|}{ Carvedilol $(n=31)$} & \multicolumn{2}{|c|}{ Nebivolol $(n=30)$} & \multicolumn{2}{|c|}{$\mathbf{P}$} \\
\hline & 3 months & 6 months & 3 months & 6 months & $\mathbf{P}^{1}$ & $\mathbf{P}^{2}$ \\
\hline NYHA class I-II & $81 \%$ & $96 \% *$ & $86 \%$ & $93 \% *$ & 0.45 & 0.54 \\
\hline HR [bpm] & $75 \pm 8$ & $67 \pm 7^{*}$ & $74 \pm 9$ & $66 \pm 6^{*}$ & 0.78 & 0.52 \\
\hline $\mathrm{SBP}[\mathrm{mm} \mathrm{Hg}]$ & $137 \pm 14$ & $122 \pm 14^{*}$ & $132 \pm 12$ & $118 \pm 15^{*}$ & 0.10 & 0.27 \\
\hline $\mathrm{DBP}[\mathrm{mm} \mathrm{Hg}]$ & $85 \pm 10$ & $75 \pm 12^{*}$ & $82 \pm 8$ & $71 \pm 9^{*}$ & 0.14 & 0.10 \\
\hline Weight [kg] & $76 \pm 17$ & $76 \pm 18^{*}$ & $73 \pm 11$ & $73 \pm 12^{*}$ & 0.43 & 0.44 \\
\hline LVEDD [mm] & $58 \pm 7$ & $57 \pm 7^{*}$ & $56 \pm 6$ & $55 \pm 5^{*}$ & 0.16 & 0.16 \\
\hline LVESD [mm] & $46 \pm 7$ & $44 \pm 7^{*}$ & $42 \pm 7$ & $41 \pm 7^{*}$ & 0.07 & 0.07 \\
\hline LVEF [\%] & $33 \pm 5$ & $36 \pm 5^{*}$ & $36 \pm 5$ & $37 \pm 5^{*}$ & 0.09 & 0.30 \\
\hline LAD [mm] & $43.4 \pm 4.4$ & $42.4 \pm 4.7$ & $41.0 \pm 3.9$ & $40.6 \pm 3.8$ & 0.26 & 0.19 \\
\hline $\mathrm{E}[\mathrm{cm} / \mathrm{s}]$ & $82 \pm 10$ & $74 \pm 1^{*}$ & $77 \pm 20$ & $69 \pm 20^{*}$ & 0.17 & 0.31 \\
\hline $\mathrm{A}[\mathrm{cm} / \mathrm{s}]$ & $89 \pm 20$ & $85 \pm 20$ & $89 \pm 10$ & $86 \pm 20$ & 0.87 & 0.81 \\
\hline$E / A$ & $0.92 \pm 0.2$ & $0.87 \pm 0.3^{*}$ & $0.86 \pm 0.2$ & $0.80 \pm 0.2^{*}$ & 0.37 & 0.30 \\
\hline DT [ms] & $196 \pm 39$ & $218 \pm 42^{*}$ & $199 \pm 34$ & $222 \pm 36^{*}$ & 0.77 & 0.71 \\
\hline IVRT [ms] & $101 \pm 12$ & $94 \pm 10^{*}$ & $98 \pm 15$ & $92 \pm 10^{*}$ & 0.43 & 0.25 \\
\hline $\mathrm{Ea}[\mathrm{cm} / \mathrm{s}]$ & $6.6 \pm 1.4$ & $6.4 \pm 1.6$ & $6.9 \pm 1.5$ & $6.8 \pm 1$ & 0.43 & 0.35 \\
\hline $\mathrm{Aa}[\mathrm{cm} / \mathrm{s}]$ & $8.1 \pm 1.7$ & $7.8 \pm 1.5^{*}$ & $8.6 \pm 1.6$ & $8.6 \pm 1.5$ & 0.28 & 0.04 \\
\hline $\mathrm{Ea} / \mathrm{Aa}$ & $0.83 \pm 0.2$ & $0.79 \pm 0.2$ & $0.78 \pm 0.2$ & $0.79 \pm 0.2$ & 0.39 & 0.99 \\
\hline $\mathrm{E} / \mathrm{Ea}$ & $12.6 \pm 2$ & $11.8 \pm 2^{*}$ & $11.5 \pm 3$ & $10.2 \pm 2^{*}$ & 0.15 & 0.01 \\
\hline NT-proBNP [pg/mL] & $445(226-845)$ & $137(113-216)^{*}$ & $395(299-661)$ & $123(105-186)^{*}$ & 0.87 & 0.41 \\
\hline
\end{tabular}

${ }^{*} p<0.05$ vs. baseline in each group; $P^{1}$ - comparisons of two groups at 3 months; $P^{2}-$ comparisons of two groups at 6 months; $A-$ mitral late diastolic velocity; DBP — diastolic blood pressure; HR - heart rate; LAD — left atrial diameter; LVEDD — left ventricular end-diastolic diameter; LVESD — left ventricular end-systolic diameter; LVEF — left ventricular ejection fraction; SBP — systolic blood pressure; other abbreviations are as shown in Table 1 and 2.

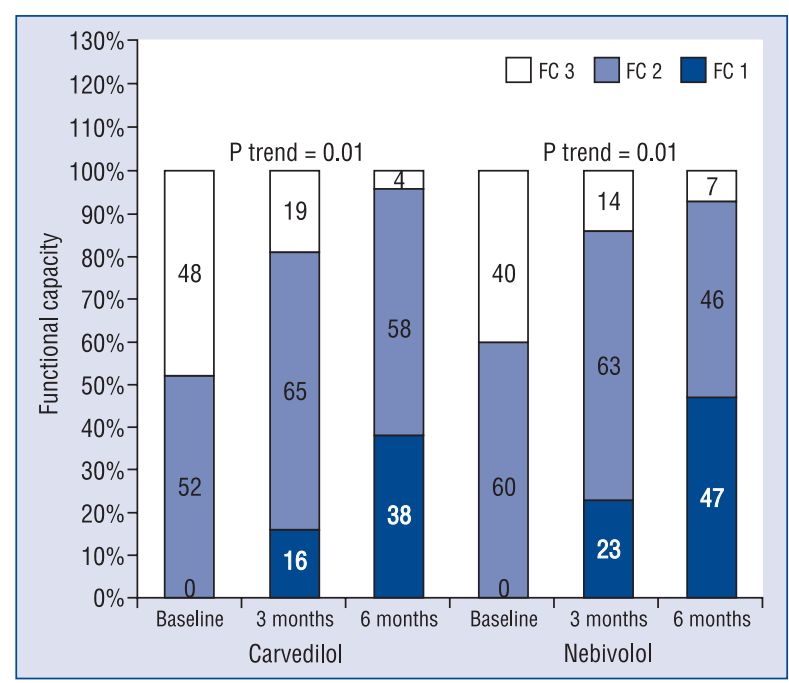

Figure 1. Functional capacity in carvedilol and nebivolol groups and its temporal changes with therapy. Although it significantly improved in each group, there was no significant difference between the two groups at 3 and 6 months ( $p>0.05)$; FC - functional capacity.

in such patients [20]. There are several explanations for their favorable effects. Beta-blockers reduce heart rate and prolong the diastolic period, thereby decreasing both oxygen consumption of myocardium and increasing oxygen supply to myocardium. Consequently, they can improve myocardial relaxation or diastolic functions and metabolism [15, 21].

Despite a low diagnostic value, mitral E/A ratio is commonly used for $\mathrm{LV}$ diastolic function in practice. Carvedilol [7, 9, 10, 18, 20, 22-24] and nebivolol $[8-10,19,25,26]$ have been reported to have different effects on mitral $\mathrm{E}$ wave velocity and $\mathrm{E} / \mathrm{A}$ ratio in previous studies. In our study, mitral $\mathrm{E} / \mathrm{A}$ ratio was similarly reduced with carvedilol or nebivolol therapy due to reduction of mitral $\mathrm{E}$ wave velocity. This finding is in accordance with the results from previous studies [10, 18-20, 22]. In contrast, Patrianakos et al. [9] have reported that mitral E/A ratio was significantly decreased with nebivolol treatment in nonischemic HF patients at 12-month follow-up, but not with carvedilol therapy.

We observed a significant improvement in DT and IVRT variables with carvediolol or nebivolol therapy, but there was no difference between the two therapy groups. Patrianakos et al. [9] have showed that DT was similarly prolonged with 
both study drugs, with earlier improvement with carvedilol. A 12-month nebivolol therapy resulted in a trend toward reduction in mitral $\mathrm{E} / \mathrm{A}$ ratio and prolongation in DT in elderly HF patients in echocardiographic substudy of SENIORS study [8]. These useful effects may be due to vasodilatory action of both agents.

In $\mathrm{HF}$ patients with low $\mathrm{EF}$, carvedilol therapy reduced mitral $\mathrm{E}$ velocity, E/A ratio and IVRT while it prolonged DT [18, 20, 22], as in our study. In contrast, mitral inflow velocities and mitral $\mathrm{E} / \mathrm{A}$ ratio remained unchanged with carvedilol therapy in $\mathrm{HF}$ patients in previous two studies $[9,10]$. Similarly, Sugioka et al. [24] have reported that carvedilol did not affect mitral $\mathrm{E}$ wave and $\mathrm{E} / \mathrm{A}$ ratio but significantly prolonged DT in patients with idiopathic dilated cardiomyopathy after 6-month therapy.

$\mathrm{E} / \mathrm{Ea}$ ratio is a reliable marker for $\mathrm{LV}$ diastolic function and well correlated with LV filling pressures [16]. Its value of $>15$ presents high LV filling pressure and has predictive value for cardiac events in patients with LV dysfunction [16, 27]. To our knowledge, there is no data about the comparative effects of carvediolol and nebivolol on $\mathrm{E} /$ Ea ratio in $\mathrm{HF}$ patients. In our study, both agents significantly reduced the $\mathrm{E} / \mathrm{Ea}$ ratio during 6 months. Although $\mathrm{E} / \mathrm{Ea}$ ratio was lower in nebivolol group than in the carvedilol group at 6 months, this difference will not provide any clinical benefit since both values are in range of 8 to 15 . In another study, carvedilol has decreased $\mathrm{E} / \mathrm{Ea}$ ratio significantly in patients with dilated cardiomyopathy secondary to muscular dystrophy [23].

Owing to the improvement in LV hemodynamics, beta-blocker therapy with an ACEI or ARB is likely to reduce NT-proBNP levels over time, but previous results are divergent [10,28-30]. These results might be due to small sample size, variable follow-up time and differences between study populations. Both carvedilol and nebivolol therapy similarly reduced NT-proBNP levels in our study at 3 and 6 months but there was no difference in neither therapy group. However, Lombardo et al. [10] have reported no reduction in NT-proBNP levels in carvediolol and nebivolol groups at 6-month follow-up.

In parallel to echocardiographic and neurohormonal improvement, we observed that both study drugs to a similar extent significantly improved functional capacity, heart rate and BP. These findings are concordant with the results from previous studies [7, 9-14, 18-20, 22, 23, 25].

\section{Limitations of the study}

There are several limitations to our study. Firstly, the small size of our study limits the statistical power since we applied strict non-inclusion criteria for the study. Secondly, we evaluated clinically the functional capacity of patients instead of exercise test or 6-minute walking distance. Also, we did not use the life-quality scoring systems. Thirdly, we would measure left atrial volume instead of its diameter. Finally, our findings reflect the situation only in patients with non-ischemic HF. It may be possible that carvedilol and nebivolol might have different effects on LV diastolic function in ischemic HF patients.

\section{Conclusions}

Our findings show that carvedilol and nebivolol have similar effects on LV diastolic functions and NT-proBNP levels in non-ischemic HF patients with reduced $\mathrm{EF}$.

\section{Conflict of interest: none declared}

\section{References}

1. Dickstein K, Cohen-Solal A, Filippatos G, McMurray JJ, Ponikowski P, Poole-Wilson PA. ESC Guidelines for the diagnosis and treatment of acute and chronic heart failure 2008: The Task Force for the Diagnosis and Treatment of Acute and Chronic Heart Failure. Eur Heart J, 2008; 29: 2388-2442.

2. Cohn JN, Levine TB, Olivari MT et al. Plasma norepinephrine as a guide to prognosis in patients with chronic congestive heart failure. N Engl J Med, 1984; 311: 819-823.

3. Rihal CS, Nishimura RA, Hatle LK, Bailey KR, Tajik AJ. Systolic and diastolic dysfunction in patients with clinical diagnosis of dilated cardiomyopathy. Relation to symptoms and prognosis. Circulation, 1994; 90: 2772-2779.

4. Parthenakis FI, Kanoupakis EM, Kochiadakis GE et al. Left ventricular diastolic filling pattern predicts cardiopulmonary determinants of functional capacity in patients with congestive heart failure. Am Heart J, 2000; 140: 338-344.

5. Cleophas TJ, van Ouwerkerk B, van der Meulen J. Nebivolol, a third generation novel beta-blocker: Systematic review. J Am Coll Cardiol, 2003; 1: 101-113.

6. Doughty RN, White HD. Carvedilol: use in chronic heart failure. Expert Rev Cardiovasc Ther, 2007; 5: 21-31.

7. Doughty RN, Whalley GA, Gamble G, MacMahon S, Sharpe N. Left ventricular remodeling with carvedilol in patients with congestive heart failure due to ischemic heart disease. Australia-New Zealand Heart Failure Research Collaborative Group. J Am Coll Cardiol, 1997; 29: 1060-1066.

8. Ghio S, Magrini G, Serio A et al. Effects of nebivolol in elderly heart failure patients with or without systolic left ventricular dysfunction: results of the SENIORS echocardiographic substudy. Eur Heart J, 2006; 27: 562-568. 
9. Patrianakos AP, Parthenakis FI, Mavrakis HE, Diakakis GF, Chlouverakis GI, Vardas PE. Comparative efficacy of nebivolol versus carvedilol on left ventricular function and exercise capacity in patients with nonischemic dilated cardiomyopathy. A 12-month study. Am Heart J, 2005; 150: 985.e9-18.

10. Lombardo RM, Reina C, Abrignani MG, Rizzo PA, Braschi A, De Castro S. Effects of nebivolol versus carvedilol on left ventricular function in patients with chronic heart failure and reduced left ventricular systolic function. Am J Cardiovasc Drugs, 2006; 6: 259-263.

11. Marazzi G, Volterrani M, Caminiti $G$ et al. Comparative long term effects of nebivolol and carvedilol in hypertensive heart failure patients. J Card Fail, 2011; 17: 703-709.

12. Packer M, Bristow MR, Cohn JN et al. The effect of carvedilol on morbidity and mortality in patients with chronic heart failure. U.S. Carvedilol Heart Failure Study Group. N Engl J Med, 1996; 334: 1349-1355.

13. Packer M, Coats AJ, Fowler MB et al. Carvedilol Prospective Randomized Cumulative Survival Study Group (COPERNICUS). Effect of carvedilol on survival in severe chronic heart failure. N Engl J Med, 2001; 344: 1651-1658.

14. Flather MD, Shibata MC, Coats AJ et al. Randomized trial to determine the effect of nebivolol on mortality and cardiovascular hospital admission in elderly patients with heart failure (SENIORS). Eur Heart J, 2005; 26: 215-225.

15. Remme WJ: Which beta blocker is most effective in heart failure? Cardiovasc Drug Ther, 2010; 24: 351-358.

16. Ommen SR, Nishimura RA, Appleton CP et al. Clinical utility of Doppler echocardiography and tissue Doppler imaging in the estimation of left ventricular filling pressures: A comparative simultaneous Doppler-catheterization study. Circulation, 2000; 102: 1788-1794.

17. Schiller NB, Shah PM, Crawford M et al. American Society Echocardiography Committee on Standards, Subcommittee on Quantitation of Two-Dimensional Echocardiograms. Recommendations for quantitation of the left ventricle by two-dimensional echocardiography. J Am Soc Echocardiogr, 1989; 2: 358-367.

18. Capomolla S, Febo O, Gnemmi M et al. Beta-blockade therapy in chronic heart failure: diastolic function and mitral regurgitation improvement by carvedilol. Am Heart J, 2000; 139: 596-608.

19. Patrianakos AP, Parthenakis FI, Mavrakis HE et al. Effects of Nebivolol on left ventricular function and exercise capacity in patients with non-ischaemic dilated cardiomyopathy. A rando- mised placebo-controlled study. Hellenic J Cardiol, 2005; 46: 199-207.

20. Tamaki S, Sakata Y, Mano T et al. Long-term beta-blocker therapy improves diastolic function even without the therapeutic effect on systolic function in patients with reduced ejection fraction. J Cardiol, 2010; 56: 176-182.

21. Wallhaus TR, Taylor M, DeGrado TR et al. Myocardial free fatty acid and glucose use after carvedilol treatment in patients with congestive heart failure. Circulation, 2001; 103: 2441-2446.

22. Palazzuoli A, Quatrini I, Vecchiato L et al. Left ventricular diastolic function improvement by carvedilol therapy in advanced heart failure. J Cardiovasc Pharmacol, 2005; 45: 563-568.

23. Rhodes J, Margossian R, Darras BT et al. Safety and efficacy of carvedilol therapy for patients with dilated cardiomyopathy secondary to muscular dystrophy. Pediatr Cardiol, 2008; 29: 343-351.

24. Sugioka K, Hozumi T, Takemoto Y et al. Early recovery of impaired coronary flow reserve by carvedilol therapy in patients with idiopathic dilated cardiomyopathy: A serial transthoracic Doppler echocardiographic study. J Am Coll Cardiol, 2005; 45: 318-319.

25. Rousseau MF, Chapelle F, Van Eyll C et al. Medium-term effects of beta-blockade on left ventricular mechanics: A double-blind, placebo-controlled comparison of nebivolol and atenolol in patients with ischemic left ventricular dysfunction. J Card Fail, 1996; 2: 15-23.

26. Nodari S, Metra M, Dei Cas L. Beta-blocker treatment of patients with diastolic heart failure and arterial hypertension. A prospective, randomized, comparison of the long-term effects of atenolol vs. nebivolol. Eur J Heart Fail, 2003; 5: 621-627.

27. Yu CM, Sanderson JE, Marwick TH, Oh JK. Tissue Doppler imaging a new prognosticator for cardiovascular diseases. J Am Coll Cardiol, 2007; 49: 1903-1914.

28. Hartmann F, Packer M, Coats AJ, et al. Prognostic impact of plasma N-terminal pro-brain natriuretic peptide in severe chronic congestive heart failure. A substudy of the study of the Carvedilol Prospective Randomized Cumulative Survival (COPERNICUS) Trial. Circulation, 2004; 110: 1780-1786.

29. Rosenberg J, Gustafsson F, Remme WJ, Riegger GAJ, Hildebrandt PR. Effect of beta-blockade and ACE inhibition on B-type natriuretic peptides in stable patients with systolic heart failure. Cardiovasc Drug Ther, 2008; 22: 305-311.

30. Li N, Li Y, Wang F et al. Does NT-proBNP remain a sensitive biomarker for chronic heart failure after administration of a beta-blocker? Clin Cardiol, 2007; 30: 469-474. 